\title{
Feasibility of HIV point-of-care tests for resource-limited settings: challenges and solutions
}

\author{
Wendy Stevens ${ }^{1,2^{*}}$, Natasha Gous ${ }^{1}$, Nathan Ford ${ }^{3}$ and Lesley E Scott ${ }^{1}$
}

\begin{abstract}
Improved access to anti-retroviral therapy increases the need for affordable monitoring using assays such as CD4 and/or viral load in resource-limited settings. Barriers to accessing treatment, high rates of loss to initiation and poor retention in care are prompting the need to find alternatives to conventional centralized laboratory testing in certain countries. Strong advocacy has led to a rapidly expanding repertoire of point-of-care tests for HIV. point-ofcare testing is not without its challenges: poor regulatory control, lack of guidelines, absence of quality monitoring and lack of industry standards for connectivity, to name a few. The management of HIV increasingly requires a multidisciplinary testing approach involving hematology, chemistry, and tests associated with the management of non-communicable diseases, thus added expertise is needed. This is further complicated by additional human resource requirements and the need for continuous training, a sustainable supply chain, and reimbursement strategies. It is clear that to ensure appropriate national implementation either in a tiered laboratory model or a total decentralized model, clear country-specific assessments need to be conducted.
\end{abstract}

Keywords: Anti-retroviral therapy, CD4, Challenges, HIV, Implementation, Point-of-care, Viral load

\section{Introduction}

Globally, the number of persons living with HIV has increased from 34 million (31.4 to 35.9 million) in 2011 to an estimated 35.3 million ( 32.2 to 38.8 million) in 2012; approximately $69 \%$ of the global HIV burden resides in sub-Saharan Africa [1]. In response to anti-retroviral therapy (ART) programs, a concurrent drop in AIDSrelated deaths from 2.3 million (2.1 to 2.6 million) in 2005 to 1.6 million (1.4 to 1.9 million) in 2012 has been recorded [1]. In order to reach the expected 2020 goals, a massive increase in HIV testing capacity will be required.

The expansion of ART programs can only be described as a huge success in low- and middle- income countries. Estimates reached 9.7 million on ART at the end of 2012, representing some $60 \%$ of those in need at that time [2]. With the new World Health Organization (WHO) guidelines changing the CD4 test threshold for

\footnotetext{
* Correspondence: wendy.stevens@nhls.ac.za

'Department of Molecular Medicine and Haematology, Faculty of Health Sciences, University of the Witwatersrand, Johannesburg, Gauteng, South Africa

${ }^{2}$ National Health Laboratory Service and National Priority Program, Johannesburg, South Africa

Full list of author information is available at the end of the article
}

treatment initiation from mid-2013, the number of individuals infected with HIV potentially requiring access to treatment has increased to an estimated 28.6 million [1]. Challenges to continued ART scale-up remain, and include improving access to HIV testing, ensuring universal access to testing, earlier initiation of treatment by improved access to HIV testing, ensuring subsequent linkage to care, and finally long-term retention in care. Each phase of HIV diagnosis and monitoring is supported by a number of tests conducted according to different algorithms in many high-burden countries, each with human and technical resource requirements. HIV rapid tests, used in adults in serial or parallel algorithms using one to three different assays, have been instrumental in ensuring wide-scale diagnosis and access to care, albeit with ongoing challenges to ensure quality. A recent estimate from President's Emergency Plan for AIDS Relief (PEPFAR) countries suggests over 80 million HIV rapid assays were performed in 2013 and that $11 \%$ of all assays were conducted as point-of-care tests (POCTs) (Jason Williams, personal communication).

CD4 testing has been the gatekeeper for assessing immune status and establishing eligibility for treatment and care. Treatment eligibility threshold levels have changed 
over time from 200 cells $/ \mu$ in 2002 [3] to 350 cells $/ \mu$ in 2010 [4]. More recently, the new consolidated WHO recommendations suggest initiation at CD4 counts of $<500$ cells $/ \mu \mathrm{l}$ [5]. Further suggestions of universal access and test and treat strategies are also being evaluated and hotly debated [6]. The latter approach is already occurring for certain high-risk population groups such as those co-infected with tuberculosis (TB), pregnant women, and children under 5 years of age. CD4 count has also been used for regular monitoring of immunological recovery on treatment, generally at six-monthly intervals. CD4 testing can be done at different tiers of the laboratory service [7] and the frequent delay in linking this assay to the initiation of patient care can result in significant loss to follow-up [8]. CD4 testing is also recommended by WHO and used in South Africa as a benchmark for establishing the risk of cryptococcal infection, where testing for cryptococcal antigen can now be done at point-of-care (POC) and the onset of meningitis can be prevented if treated with fluconazole [9].

The HIV viral load (VL) assay, a nucleic-acid-based test, is used to monitor response to treatment; an undetectable viral load defines treatment success. VL testing is frequently done in centralized facilities and currently requires expensive instrumentation, technical skill, and has relatively high costs per assay. Despite these challenges, this assay has gained its rightful place in guidelines and clinical practice and is thought to be the most reliable marker for treatment success $[10,11]$. The development pipeline of POC VL assays promises to deliver a number of options to improve access and facilitate earlier identification of treatment failure. This will allow clinicians to avoid premature switching of regimens, particularly in regions with limited drug availability, potentially improving patient adherence and reducing the development of drug resistance [12]. Also, the percentage of failures using this assay can provide a monitor of both individual and program success [13]. As access to VL testing is improving, the role of CD4 measurements is being reassessed. Numerous studies have demonstrated that for the vast majority of people living with HIV who are receiving ART and are virally suppressed, CD4 cell count does not decline over time [14]. Other studies have shown that one third of individuals whose CD4 count was greater than 350 cells/ $\mu$ l had viral loads greater than 100,000 HIV RNA copies/ml [15]. A metaanalysis of seven studies assessing the accuracy of clinical or CD4 tests in predicting virological failure found a poor sensitivity of $26.6 \%$ and a positive predictive value of $49.4 \%$ [11]. This suggests that in situations where viral load is available routinely, CD4 monitoring can be reduced in frequency or stopped altogether. Recognizing this opportunity to save resources, the South African ART guidelines in 2013 recommended stopping routine
CD4 monitoring in people who are stable on ART and a number of other countries are considering moving in this direction [16].

In addition to the core assays described in individuals with HIV, there are also hematology and biochemistry assays that remain important, including hemoglobin, creatinine (especially for tenofovir initiation) and liver transaminase tests as well as assays for the diagnosis of opportunistic infections such as TB and cryptococcal infection. The diagnosis and treatment of TB is critical in low- and middle- income countries where a significant proportion of individuals with HIV infection are co-infected with TB. In South Africa as an example, co-infection rates are as high as $65 \%$ to $70 \%$ [17].

To address all the needs described above and in the face of the successes of rapid tests such as those for HIV, malaria and, more recently, cryptococcal antigen, there is a drive now towards using POCT for the noncommunicable diseases such as diabetes, cardiovascular disease and cancer, many of which are associated with long-term management of people living with HIV. Thus, there is an expanded list of multidisciplinary testing needs at primary health clinics (PHC). Performing and interpretation of these tests will potentially require significantly more expertise than a single rapid HIV antibody test.

\section{History of point-of-care testing}

POCT is an old approach to testing that has been around for decades and remains as controversial today as it was when first introduced. POCT refers to testing that is performed near to or at the site of patient care, with the result leading to a possible or immediate change to patient care [18]. The rationale is largely based on a need for shortening the time to decision making. The literature provides a myriad of different definitions such as the Clinical Laboratory Standards Institute in the USA, which defines the purpose of POCT being the provision of timely results that clinically and cost-effectively contribute to management decisions [19]. The first references to POCT date back to the early 1990s and focused largely on glucose testing for diabetes and blood gas analyzers in ICUs and operating theaters [20]. The controversy around managerial, quality and regulatory ownership remains a problem and it has been suggested that this is still a 'work in progress' [21]. Despite this, POCT is the fastest growing segment of the diagnostic industry (10\% to $14 \%$ annually), accounting for one in four tests within the developing world $[22,23]$. A recent review reported that POCT accounts for $25 \%$ of total laboratory revenue [24]. New diagnostics into which POCT has expanded include cardiac markers, coagulation assays, substance abuse and homebased HIV testing, to name a few [25]. Interestingly, POC devices include not only ex vitro but also in vitro and 
in vivo methodologies (continuous monitoring devices) [26]. Technological advances such as microfluidics [27], miniaturization [28], microfabrication, simple power and affordable light sources, electromagnetic actuation of fluids using microelectronics and, more recently, nanodiagnostics $[29,30]$ have facilitated the development of more complex assays capable of placement at the POC [28]. Thus, rapid tests described for HIV diagnosis have been described as first-generation POC assays, involving antigens and antibodies and simple biochemistry and hematology; the second generation is infinitely more complex and based on cell detection or nucleic acid amplification and detection; the third generation will involve complex analyzers that could have multiplexing capabilities [31].

\section{Global perspective on point-of-care testing}

The unmet laboratory needs for assays to address communicable diseases such as HIV, TB and malaria appear to have assisted in catalyzing the POC diagnostics industry as a whole. Both communicable and non-communicable diseases will in future reap the benefits as appropriate implementation strategies are developed [31]. This is particularly important when predictions for the future suggest that diabetes may well be a more important risk factor for TB than HIV. Global market assessments have suggested that the increase in diabetes and thus glucose testing comprises at least $10 \%$ of the global POCT market [32]. The growth in POC HIV testing has been further reinforced by strong advocacy from groups such as the WHO (One pillar of Treatment v2.0 \{WHO Department of HIV/AIDS, 2011 \#99\}guideline [33], WHO 2013 treatment guidelines [34]), UNITAID (market catalysts; Geneva), the Bill and Melinda Gates Foundation, the Clinton Foundation, PEPFAR and the African Society of Laboratory Medicine, who have been tasked with promoting guidance and implementation in field sites. This drive has begun to address many of the factors mentioned above, such as the absence of laboratories or access to assays such as CD4 and VL testing for the diagnosis and monitoring of HIV in remote sites. Alternatives to conventional centralized testing are being driven by the high rates of loss to initiation for both HIV and TB, as well poor retention in care [35]. These activities have catalyzed funders, suppliers, users and patients in galvanizing the POC diagnostics industry into action. In addition, POCT has been incorporated into the Global Health Strategy on HIV/AIDS [36]. Both the WHO and the London School of Tropical Medicine and Hygiene have been tasked with bringing forward multi-center laboratory-based validations of POC assays followed by an evaluation of their implementation in the field [37]. Strong emphasis has also been placed on the need for monitoring the impact and cost of the interventions across the entire continuum of care. By nature of the low throughput of these technologies and the additional human resources required in the field for testing and maintenance, the total assay costs can be as expensive, or more expensive, than laboratory testing. A strong emphasis needs to be placed on innovative strategies to ensure quality for tests that are being conducted in volumes far beyond that covered by conventional laboratory quality assurance plans and accreditation status. In South Africa, there is an ISO standard (ISO22789) that has been implemented for accredited laboratories to follow if they are conducting and supporting POC testing [38]. Perhaps a similar approach to accreditation of clinic sites conducting POC testing with a simpler standard and checklist could be used to ensure quality is maintained in fieldtesting sites.

\section{The pipeline for HIV diagnosis and monitoring}

There is an ever-expanding pipeline associated with the strong advocacy for POCT from global players, who maintain that universal access for HIV and TB care requires the use of POCT for earlier testing and improved retention in care. Cited advantages of POCT include improved turnaround time, greater accessibility, potentially improved patient retention and possible reduction in overall healthcare costs. However, despite the rapid growth and interest in POCT, many aspects remain controversial, in part because this approach challenges the conventional approach to laboratory testing, which remains the prevailing paradigm in many countries. In addition, while numerous early or near market entry products are available, at the time of writing few could be purchased on a large scale, outside of rapid HIV and malaria strip-based tests, and a monopoly exists of one or two suppliers with a proven track record for CD4 testing, such as the PIMA assay (Alere Inc., Waltham, MA, USA). In the VL arena, many early market entry products are available and development has been heavily funded, yet only three were available for clinical validation as of April 2014 - the LIAT $^{\text {тм }}$ Analyzer (IQuum, Inc., Roche) [39], Alere ${ }^{\mathrm{Tm}}$ q HIV-1/2 Detect (Alere) [40] and Samba (Diagnostics for the Real-World, Ltd.) [41] - and manufacturing track records for scale-up were not available. The upcoming pipeline for HIV CD4 and VL testing with their performance characteristics are summarized in the landscape document produced annually by UNITAID [12]. A plethora of fast followers are in various stages of research, development and evaluation and include the MBio POC CD4 (MBio Diagnostics, Inc)(Co,USA) [42], Daktari CD4 Counter (Daktari Diagnostics, Inc.)(MA, USA), FACSPresto ${ }^{\mathrm{Tm}}$ (BD Biosciences)(NJ, USA) [43], Visitect ${ }^{\circ}$ (Omega Diagnostics), Zyomyx CD4 (Zyomyx, Inc.) and EMD Millipore ${ }^{\oplus}$ Muse $^{\text {Im }}$ (Merck)(Darmstadt,Germany) [12]. For VL testing, these include the GeneXpert ${ }^{\oplus}$ Viral Load system (Cepheid, Sweden), the EOSCAPE-HIV ${ }^{\mathrm{s}}$ Rapid RNA Assay system 
(Wave 80 Biosciences)(CA,USA) [44], TrueLab ${ }^{\text {TM }}$ Real Time micro PCR system (MolBio Diagnostics, Ltd.), Goa, India Savanna VL test and platform (Northwestern Global Health Foundation in collaboration with Quidel Corporation) and Bioluminescent Assay in Real Time technology (Lumora, Ltd.)(cambridgeshire,UK) [45], amongst others [12].

In countries where significant laboratory infrastructure currently exists in both the public and private sectors, the sheer volumes of testing may make total decentralization prohibitive in terms of instrumentation and human resource requirements. In these instances, POC assays may and do have a role to play where gaps in service are noted, which can be identified by approaches such as geographic information systems mapping to ensure a national 'total coverage model'. The total coverage model is a new term being used in laboratory testing circles which refers to a tiered implementation model that includes both POC testing and different tiers of laboratory testing to ensure access for the entire national population. POCTs are also used heavily in specific niche areas such as hemoglobin in emergency rooms or renal clinics. A particular niche for the VL assay could, for example, be in maternity wards and antenatal care clinics where pregnant women infected with HIV could be monitored for risk of transmission and success of treatment strategies, and exposed infants could be tested at birth for HIV and then treatment initiated as soon as possible.

Major issues surrounding the implementation of POCT exist and include poor regulatory control, difficulties in ongoing monitoring of quality, and limited availability of guideline documents for the safe implementation of POC devices. In addition, there are few studies that report data on full economic costing for POC [46], which is likely to vary depending on tests used, diseases investigated and model input parameters.

There is a dearth of well-designed randomized controlled clinical trials (RCTs) to evaluate the outcomes and impact of the implementation of POCT. Most notable for their contributions to the POC literature are a group led by Shephard in Australia [47,48]. Although evaluating other assays in a general practitioner setting in Australia, the final study conclusions were that POCT was not inferior to laboratory-based testing, but came at a substantially higher cost that needs to be weighed against overall health benefits. Various clinical experiences were presented at a recent forum held in South Africa, with a number of studies reporting progress in RCT studies such as the Home-based Care Plus trial in Kwazulu-Natal, Rapid Initiation of Anti-retrovirals in Pregnancy (RAP) study in Cape Town, the Grand Challenges Canada RCT, and RapIT (Midrand PHC, South Africa). Results are still awaited eagerly and will help form policy but have shown clearly that POCT is just one step in a multi-step process along the continuum of care [49]. Other experiences show that POCT has great potential for certain high-risk populations such as migrants or adolescents where loss to follow-up is high and where immediate results would add value [49].

Pilot studies on the implementation of PIMA CD4 POC testing in South Africa and Mozambique have demonstrated that time to initiation is reduced; however, challenges were identified in that nurses perceived POC implementation as additional workload, and patients migrated from facilities before staff were able to track, record and file the results in patients' folders [49]. Experiences from Mozambique showed that after the introduction of POC CD4, the loss to follow-up before CD4 staging dropped, ART initiation rate increased, and time to ART initiation was reduced from 48 days to 20 days [50]. Retention rates in care, however, remained the same. It was recommended by this group that deploying POC should be done in in conjunction with conventional testing as part of a total laboratory network and there was acknowledgement that POC testing is far from error-proof. Only 20\% of Mozambique's CD4 counts are conducted at POC. High invalid rates were noted using POC CD4 tests in this study. The authors warned that simple implementation is not always efficient - access does not necessarily mean that the patient gets care (approximately $25 \%$ of patients did not get CD4 testing even with POCT on site). They also highlighted that significant health systems strengthening is needed and clinic workflow re-engineering. A meta-analysis of the performance of PIMA is underway and preliminary analysis have revealed that the performance of the instrument on venous specimens is as good as current gold standard technology. However, the performance on capillary-derived specimens showed increased variability at the 350 cells/ $\mu$ l threshold, resulting in higher false-positive rates that would lead to more patients being placed on ART (unpublished results, Lesley Scott personal communication).

\section{Approaches to ensuring quality testing}

The US Food and Drug Administration requirements for defining a simple test are that it should be rapid, easy to perform, require minimal training and no specialized laboratory setup, and reagents should be stable and temperature independent. However, few assays actually meet these requirements. It should be noted that assay transfer from the laboratory to POC is not synonymous with improved quality of care. Implementation at the POC will require facilitation in a step-wise fashion with careful monitoring and evaluation at each step. The approach to quality of rapid lateral flow-based assays will be different to those that are device based. Several guidelines for 
HIV rapid testing have been written over the years, but uptake of these recommendations has been poor in most resource-limited settings. In fact, many of these assays are considered Clinical Laboratory Improvement Amendment-waived because they are simple tests with a low risk for an incorrect result and are thus not quality assured in developed countries such as the USA.

While programs such as the WHO pre-qualification process [51] have provided guidance by conducting product and supplier evaluations and validations, and the Centers for Disease Control and Prevention (CDC) has done similar work for PEPFAR-related programs, there is a need for harmonization of approaches and standardization of protocols with greater co-operation between stakeholders. There needs to be co-ordination between and a review of all strategies and guidelines so that a simple, single guidance can be provided for countries. Quality needs to be addressed, within the laboratory and at the pre-analytic, analytical and postanalytical phases [22]. For rapid assays, the sheer volumes of assays conducted make conventional internal and external quality approaches extremely difficult to implement. Strategies employed have included the use of external quality assurance (EQA) material using dried tube spots for various HIV rapid assays [52] or dried culture spots for near POCT for TB [53,54]. Innovative strategies are required for material distribution and data collection across large programs. Regular training and re-training, competency assessments, and ongoing supervision and mentoring of staff conducting assays are all critical to ensuring continuous maintenance of quality.

For device-based assays, an approach that is under scrutiny is the use of real-time continuous monitoring using various connectivity systems linked to analyzers in the field $[55,56]$. Connectivity provides a means not only to ensure analyzer performance meets requirements, but also of collecting programmatic data, distribution of results and identifying the need to intervene should problems arise. Data ownership and data security are issues that need to be addressed. Each analyzer, however, frequently connects to the middleware or software solution via a different mechanism and there is thus a need for industry standards for POCT connectivity [57]. Several middleware programs have been evaluated that link to laboratory information systems in South Africa with success, although approaches differ in different regions depending on wireless availability, internet access and computer literacy. Thus solutions may need to be contextualized within different geographic regions. Simpler approaches may include the use of bi-directional short message service printers with additional capabilities for data collection and acknowledgement of receipt of results [58]. To improve retention in care, patients can be recalled for results, and this makes for a reasonably successful means of improving adherence [59]. The role of secondary and tertiary laboratories in the management of quality in PHCs is essential and many believe POCT should be a natural extension of the laboratory [60].

Supply chain management and procurement strategies need to be well planned. Global procurement and global forecasting may play a larger role than for other assays because the production lines for new assays entering the market are frequently unable to meet the demand of rapid recommendations that lead to rapid global uptake. Engagement with industry in the pre-market phase may help to ensure quality features are built into the system, connectivity is considered, and production meets the needs based on information provided on disease prevalence and likely test numbers. UNITAID, as an organization that funds approaches to catalyze and effect market changes, can stimulate additional approaches improving access. Advocacy for quality assured, appropriately selected assays used in settings where impact can be demonstrated is strongly needed.

\section{Ownership and accountability}

There is a general consensus that ownership should be at the level of in-country ministries of health. A POCT policy needs to be embedded within national strategic laboratory plans, the development of which was strongly advocated for by the Maputo declaration [61]. A single strategic national plan for the introduction of POCT in a country is likely to solicit donor funding or that of local treasuries in a far more effective manner. It is imperative that technical task teams are established to support decision making. The composition of the team should include clinicians; laboratorians; health economists; procurement, supply and distribution workers; and funders. Strong partnerships with industry need to be facilitated because the ongoing procurement, maintenance of analyzers and product failures need to be addressed. As a result of recent product failures in the HIV arena impacting many countries, a task team was established with expertise from organizations such as the WHO, CDC and other partners. This may be useful going forward to urgently address product failures as this body is formalized. This brings in the concept of a far more active reporting to support post-market surveillance, currently poorly coordinated the world over. Ownership of the POCT process, however, needs to extend to users of the assays and the communities that are tested, with creative ways developed for incentivizing healthcare workers conducting the tests to maintain high quality standards.

\section{Conclusions}

POCT will improve access to needed HIV and associated diagnostics, but these assays are not without limitations 
that should be noted and reported. There is a need to integrate these technologies cost-effectively and efficiently into clinical algorithms and existing laboratory networks. In costing, it should be emphasized that context matters, particularly human resources and test volumes. There is much to be done in this field. Notably, large randomized studies measuring the impact of a diagnostic intervention along the entire continuum of care are currently an exception and need to be encouraged and supported. Standardization of assay evaluation and development of appropriate internal and external quality control are important activities that need support. Regulatory hurdles need to be overcome and developed in many countries. Global harmonization of all stakeholder activities is essential to get the product from an idea to the bench and ultimately to the patient bedside. The likelihood is that in many countries POCT will be strategically deployed in a hybrid model with support from the conventional tiers of in-country laboratories.

\section{Abbreviations}

ART: antiretroviral therapy; CDC: Centers for Disease Control and Prevention; EQA: external quality assurance; PEPFAR: President's Emergency Plan for AIDS Relief; PHC: primary health-care clinic; POC: point-of-care; POCT: point-of-care testing; RCT: randomized control trial; TB: tuberculosis; VL: viral load; WHO: World Health Oganization.

\section{Competing interests}

The authors declare that they have no competing interests.

\section{Authors' contributions}

WS wrote the first draft of the manuscript. LS and NG did a review of the manuscript; both are involved in POC projects in the National HIV/TB program in South Africa and provided information. NG assisted with sourcing references. NF reviewed the document. All authors have read and approved the final manuscript.

\section{Authors' information}

WS is currently Professor and Head of the Department of Molecular Medicine and Haematology at the University of the Witwatersrand; and the National Health Laboratory Service (NHLS) from 2003 to current. Her research efforts have been largely focused in HIV for the past 12 years and this can be supported by over 170 peer-reviewed publications and 140 conference presentations. She has contributed significantly to the development of capacity for affordable, accessible HIV diagnosis and monitoring in South Africa and over 60 centers in sub-Saharan Africa. Research activities have included the expansion of early infant diagnosis of HIV, affordable viral load, CD4 and investigation of HIV drug resistance. Since November 2010, she has been appointed head of National Priority Programs at the NHLS focusing on laboratory efforts related to HIV and TB. Her current roles include National Rollout of GeneXpert technology across microscopy centers in South Africa. Her current work is the evaluation and validation of POCT, both instruments and positioning thereof within the healthcare system in South Africa. NG is a PhD student, currently holding the position of Medical Scientist in the Research and Development Unit in the Department of Molecular Medicine and Haematology at the University of the Witwatersrand. Her main areas of research include the development and evaluation of novel, rapid and affordable HIV and TB diagnostic assays, particularly for use in low-resource settings. NG is part of the POC Research Group established by the NHLS National Priority Program to investigate integration of HIV/TB services at POC and was the R\&D scientist involved in the development and production of an EQA and verification program for the NHLS National GeneXpert rollout program (under LS)
NF has worked on improving access to HIV/AIDS treatment and care in resource-limited settings for the last 15 years, with a particular focus on sub-Saharan Africa.

LS is an applied scientist in the Department of Molecular Medicine and Haematology, and has for the past 15 years focused on designing, developing, evaluating and implementing laboratory diagnostic technologies for individuals infected with HIV and TB. She has over 50 publications, more than 100 abstracts at local and international conferences, and is a reviewer for several journals and part of editorial boards within her field. Her more recent innovation is the development of a novel quality monitoring system for the Gene Xpert MTB/RIF test using dried culture spots of inactivated Mycobacterium tuberculosis. These developments together with three other WITS patents and one trademark emphasize the contribution of LS's research to improving healthcare in South Africa.

\section{Acknowledgements}

The knowledge-base was attained through work done with funds received from Grand Challenges Canada POC implementation grant (grant 0007-02-01-01-01).

\section{Author details}

'Department of Molecular Medicine and Haematology, Faculty of Health Sciences, University of the Witwatersrand, Johannesburg, Gauteng, South Africa. ${ }^{2}$ National Health Laboratory Service and National Priority Program, Johannesburg, South Africa. ${ }^{3}$ Department of HIV/AIDS, World Health Organization, Geneva, Switzerland.

Received: 19 May 2014 Accepted: 11 August 2014

Published: 8 September 2014

\section{References}

1. UNAIDS: Global Report. UNAIDS Report on the global AIDS epidemic 2013. [www.unaids.org/en/resources/campaigns/globalreport2013/ globalreport/]

2. UNAIDS: Global Report. UNAIDS Report on the global AIDS epidemic 2012. [http://www.unaids.org/en/resources/publications/2012/name,76121, en.asp]

3. World Health Organization: Scaling up antiretroviral therapy in resource limited settings. Guidelines for a public health approach 2002. [http://data.unaids.org/publications/IRC-pub01/jc354scalingupguidelinesdoc_en.pdf]

4. World Health Organization: Antiretroviral therapy for HIV infection in adults and adolescents: Recommendations for a public health approach 2010. [http://whqlibdoc.who.int/publications/2010/9789241599764_eng.pdf]

5. World Health Organization: Consolidated guidelines on the use of antiretrovirals for treating and preventing HIV infection: Recommendations for a public health approach 2013. [http://apps.who.int/iris/bitstream/ 10665/85321/1/9789241505727_eng.pdf?ua=1]

6. De Cock KM, El-Sadr WM: Perspective: When to start ART in Africa-an urgent research priority. N Engl J Med 2013, 368:886-889.

7. Nkengasong JN: A shifting paradigm in strengthening laboratory health systems for global health: acting now, acting collectively, but acting differently. Am J Clin Pathol 2010, 134:359-360.

8. Clouse K, Pettifor AE, Maskew M, Bassett J, Van Rie A, Behets F, Gay C, Sanne I, Fox MP: Patient retention from HIV diagnosis through one year on antiretroviral therapy at a primary health care clinic in Johannesburg, South Africa. J Acquir Immune Defic Syndr 2013, 62:e39-e46.

9. Govender N, Chetty V, Roy M, Chiller TM, Oladoyinbo S, Maotoe T, Stevens WS, Pinini Z, Spencer D, Venter F, Jassat W, Cameron D, Meintjes G, Mbengashe T, Pillay $Y$ : Phased implementation of screening for cryptococcal disease in South Africa. S Afr Med J 2012, 102:914-917.

10. Calmy A, Ford N, Hirschel B, Reynolds SJ, Lynen L, Goemaere E, Garcia de la Vega F, Perrin L, Rodriguez W: HIV viral load monitoring in resource-limited regions: optional or necessary? Clin Infect Dis 2007, 44:128-134.

11. Rutherford GW, Anglemyer A, Easterbrook PJ, Horvath T, Vitoria M, Penazzato M, Doherty MC: Predicting treatment failure in adults and children on antiretroviral therapy: a systematic review of the performance characteristics of the 2010 WHO immunologic and clinical criteria for virologic failure. AIDS 2014, 2:S161-S169. 
12. World Health Organization/UNITAID: HIV/AIDS Diagnostics Technology Landscape 2014 4th Edition. [http://www.unitaid.eu/images/ marketdynamics/publications/UNITAID-HIV_Diagnostic_Landscape4th_edition.pdf]

13. World Health Organization/UNITAID: HIV/AIDS: Diagnostic Technology landscape. Semi-annual update 2013. [http://www.unitaid.org/images/ UNITAID_2013_Semi-annual_Update_HIV_Diagnostics_Technology_ Landscape.pdf]

14. Gale HB, Gitterman SR, Hoffman HJ, Gordin FM, Benator DA, Labriola AM, Kan VL: Is frequent CD4+ T-lymphocyte count monitoring necessary for persons with counts $>=300$ cells/uL and HIV-1 suppression? Clin Infect Dis 2013, 56:1340-1343.

15. Mosen DM, Horberg M, Roblin D, Gullion CM, Meenan R, Leyden W, Hu W: Effect of once-daily FDC treatment era on initiation of CART. HIV AIDS (Auckl) 2010, 2:19-26.

16. World Health Organization: March 2014 Supplement to the 2013 Consolidated Guidelines on the use of Antiretroviral Drugs for Treating and Preventing HIV infection. [http://www.who.int/hiv/pub/guidelines/ arv2013/arvs2013upplement_march2014/en/]

17. World Health Organization: Global Tuberculosis Report 2013. Country profiles. [http://www.who.int/tb/publications/global_report/en/

18. Kost GJ: Point-of-care testing in intensive care, and planning and implementing point-of-care test systems. In Principles and Practice of Intensive Care Monitoring. Edited by Tobin MA. New York, NY: McGraw Hill; 1998:1267-1328.

19. Zucker ML: Point of care testing guideline published by CLSI. Lab Medicine 2010, 41:499-500

20. Kilgore ML, Steindel SJ, Smith JA: Cost analysis for decision support: the case of comparing centralized versus distributed methods for blood gas testing. J Healthc Manag 1999, 44:207-215.

21. Kost GJ, Ehrmeyer SS, Chernow B, Winkelman JW, Zaloga GP, Dellinger RP, Shirey T: The laboratory-clinical interface: point-of-care testing. Chest 1999, 115:1140-1154.

22. Plebani M: Does POCT reduce the risk of error in laboratory testing? Clin Chim Acta 2009, 404:59-64.

23. Warsinke A: Point-of-care testing of proteins. Anal Bioanal Chem 2009, 393:1393-1405.

24. Hammett-Stabler CA, Nichols JH: Point-of-care testing, a critical component of laboratory medicine. Clin Biochem 2009, 42:135.

25. Lewandrowski K: Point-of-care testing: an overview and a look to the future (circa 2009, United States). Clin Lab Med 2009, 29:421-432.

26. Girardin CM, Huot C, Gonthier M, Delvin E: Continuous glucose monitoring: a review of biochemical perspectives and clinical use in type 1 diabetes. Clin Biochem 2009, 42:136-142.

27. Sorger PK: Microfluidics closes in on point-of-care assays. Nat Biotechnol 2008, 26:1345-1346.

28. Kricka LJ: Microchips, microarrays, biochips and nanochips: personal laboratories for the 21st century. Clin Chim Acta 2001, 307:219-223.

29. Alharbi KK, Al-Sheikh YA: Role and implications of nanodiagnostics in the changing trends of clinical diagnosis. Saudi J Biol Sci 2014 21:109-117.

30. Syed MA: Advances in nanodiagnostic techniques for microbial agents. Biosens Bioelectron 2014, 51:391-400.

31. Jani IV, Peter T: How point-of-care testing could drive innovation in global health. New Engl J Med 2013, 368:2319-2324.

32. Grand View Research: Report 2014: Point of Care Diagnostics (POC) Market Analysis By Product (Blood Gas/Electrolytes, Infectious Diseases, Hospital Glucose Testing, Cardiac Markers, Hematology, Coagulation, Primary Care Systems, Decentralized Clinical Chemistry, Urinalysis, Drug Abuse Testing, Fertility, OTC and Rapid Diagnostic Tests) And Segment Forecasts To 2020. [http://www.grandviewresearch.com/industry-analysis/ point-of-care-poc-diagnostics-industry]

33. WHO Department of HIV/AIDS,UNAIDS: The treatment 2.0 framework for action: catalysing the next phase of treatment, care and support. www.who.int/hiv/pub/arv/treatment/en/ Accessed August 2014.

34. WHO: Consolidated guidelines on the use of antiretroviral drugs for treatment and prevention of HIV infection. www.who.int/hiv/pub/ guidelines/arv2013/en/. Accessed August 2014

35. Fox MP, Shearer K, Maskew M, Macleod W, Majuba P, Macphail P, Sanne I: Treatment outcomes after 7 years of public-sector HIV treatment. AIDS 2012, 26:1823-1828.
36. WHO: Global Health Strategy on HIV/AIDS: 2011-2015. www.who.int/hiv/ pub/hiv_strategy/en/. Accessed August 2014

37. Peeling RW, McNerney R: Emerging technologies in point-of-care molecular diagnostics for resource-limited settings. Expert Rev Mol Diagn 2014, 14:525-534.

38. South African National Accreditation System: ISO Point-of-care testing (POCT) - requirements for quality and competence, in ISO 22870:2006200. [http://www.iso.org/iso/catalogue_detail.htm?csnumber=35173]

39. Scott L: An evaluation of the Liat assay (IQUUM). [as/m.org/aslm2012/ images/docs/Friday-December-7th-2012/Oral-Presentations/Point-of-Care\% 20Diagnostics\%20-\%20Evaluation\%200utcomes/3.\%20lesley\%20scott.pdf]

40. Jani IV, Meggi B, Mabunda N, Vubil A, Sitoe NE, Tobaiwa O, Quevedo J, Lehe JD, Loquiha O, Vojnov L, Peter TF: Accurate early infant HIV diagnosis in primary health clinics using a point-of-care nucleic acid test. J Acquir Immune Defic Syndr 2014, 16:e1-e4.

41. Lee H, Diagnostics for the Real World, Ltd: Point of care HIV molecular tests: Bridging the gap between laboratory and patient. In Presentation in 1st International Conference of the African Society for Laboratory Medicine: 2012 December 1-7; Cape Town, South Africa. Cape Town: ASLM; 2013.

42. Logan C, Givens M, Ives JT, Delaney M, Lochhead MJ, Schooley RT, Benson CA: Performance evaluation of the MBio Diagnostics point-of-care CD4 counter. J Immunol Methods 2013, 387:107-113.

43. Bornheimer $\mathrm{S}$, Bui $\mathrm{N}$, Le $\mathrm{D}$, Wai $\mathrm{H}$, Tran A, Goldberg E, Bouic $\mathrm{P}$, Huang W, Sugarman J, Crowe M, Kumar V, Shook J, Tomas C, Yu J, Clark A, Broszeit E, Chen R, Bush-Donovan C: Development of the BD FACSPresto instrument, a new point-of-care system for CD4+ counting. In Poster presentation TUPE265 in 7th IAS Conference on HIV Pathogenesis, Treatment and Prevention: 2013 June 30 July 3, Kuala Lumpur. Kuala Lumpur: International AIDS Society; 2013.

44. Murtagh M: Viral load: Current technologies and the pipeline, including point-of-care assays. [http://www.aslm.org/?wpdmdl=95]

45. Gandelman OA, Church VL, Moore CA, Kiddle G, Carne CA, Parmar S, Jalal H, Tisi LC, Murray JA: Novel bioluminescent quantitative detection of nucleic acid amplification in real-time. PLoS One 2010, 5:e14155.

46. Laurence C, Gialamas A, Yelland L, Bubner T, Ryan P, Willson K, Glastonbury B, Gill J, Shephard M, Beilby J: A pragmatic cluster randomised controlled trial to evaluate the safety, clinical effectiveness, cost effectiveness and satisfaction with point of care testing in a general practice setting - rationale, design and baseline characteristics. Trials 2008, 9:50.

47. Shephard MD, Mazzachi BC, Watkinson L, Shephard AK, Laurence C, Gialamas A, Bubner T: Evaluation of a training program for device operators in the Australian Government's Point of Care Testing in General Practice Trial: issues and implications for rural and remote practices. Rural Remote Health 2009, 9:1189.

48. Shephard MD: Influence of geography on the performance of quality control testing in the Australian Government's point of care testing in general practice trial. Clin Biochem 2009, 42:1325-1327.

49. South African National Department of Health, National Health Laboratory Service: Point-of-care testing: Position paper Forum Report to support National Strategic Plan for POCT for the management of HIV and TB in South Africa: An overview of the Point-Of Care Forum hosted by the National Department of Health. 2013. In preparation.

50. Jani IV, Sitoe NE, Alfai ER, Chongo PL, Quevedo Jl, Rocha BM, Lehe JD, Peter TF: Effect of point-of-care CD4 cell count tests on retention of patients and rates of antiretroviral therapy initiation in primary health clinics: an observational cohort study. Lancet 2011, 378:1572-1579.

51. World Health Organization: Prequalification of diagnostics. [http://www. who.int/topics/prequalification/en/]

52. Benzaken AS, Bazzo ML, Galban E, Pinto IC, Nogueira CL, Golfetto L, Benzaken NS, Sollis KA, Mabey D, Peeling RW: External quality assurance with dried tube specimens (DTS) for point-of-care syphilis and HIV tests: experience in an indigenous populations screening programme in the Brazilian Amazon. Sex Transm Infect 2014, 90:14-18.

53. Gous N, Cunningham B, Kana B, Stevens W, Scott LE: Performance monitoring of mycobacterium tuberculosis dried culture spots for use with the GeneXpert system within a national program in South Africa. J Clin Microbiol 2013, 51:4018-4021.

54. Scott LE, Gous N, Cunningham BE, Kana BD, Perovic O, Erasmus L, Coetzee GJ, Koornhof H, Stevens W: Dried culture spots for Xpert MTB/RIF external quality assessment: results of a phase 1 pilot study in South Africa. J Clin Microbiol 2011, 49:4356-4360. 
55. Cepheid: Remote connectivity. In Presentation in 5th Annual GLI Conference: 2013 April 15-18; Annecy, France. [http://www.gxalert.com/ index.php/presentation-at-the-5th-annual-gli-conference-in-annecy-franceapril-15-18-2013/]

56. Kessler HH, Jungkind D, Stelzl E, Direnzo S, Vellimedu SK, Pierer K, Santner B, Marth E: Evaluation of AMPLILINK software for the COBAS AMPLICOR system. J Clin Microbiol 1999, 37:436-437.

57. St John A, Merkle H: A connectivity standard for point-of-care testing. Med Device Technol 2001, 12:23-26.

58. Stevens W: Viral load testing in Africa - 23 years later. [www.aslm.org/? wpdmdl=85]

59. Faal M, Naidoo N, Glencross DK, Venter WD, Osih R: Providing immediate CD4 count results at HIV testing improves ART initiation. J Acquir Immune Defic Syndr 2011, 58:e54-e59.

60. Manyazewal T, Paterniti AD, Redfield RR, Marinucci F: Role of secondary level laboratories in strengthening quality at primary health care facilities' laboratories: an innovative approach to ensure accurate HIV tuberculosis and malaria tests is resource-limited settings. Diagn Microbiol Infect Dis 2013, 75:55-59.

61. World Health Organization: The Maputo declaration on strengthening of laboratory systems 2008. [http://www.who.int/diagnostics_laboratory/ Maputo-Declaration_2008.pdf]

doi:10.1186/s12916-014-0173-7

Cite this article as: Stevens et al:: Feasibility of HIV point-of-care tests for resource-limited settings: challenges and solutions. BMC Medicine 2014 12:173

\section{Submit your next manuscript to BioMed Central and take full advantage of:}

- Convenient online submission

- Thorough peer review

- No space constraints or color figure charges

- Immediate publication on acceptance

- Inclusion in PubMed, CAS, Scopus and Google Scholar

- Research which is freely available for redistribution 